\title{
Fish-oil consumption is inversely correlated with depression and cognition decline in healthy Irish elderly adults
}

\author{
E. M. O'Connor ${ }^{1}$, S. E. Power ${ }^{1}$, G. F. Fitzgerald ${ }^{1,2}$ and P. W. O’Toole ${ }^{1,2}$, \\ On Behalf Of The Eldermet Consortium (see http://eldermet.ucc.ie For Consortium Membership) \\ ${ }^{1}$ Department of Microbiology and ${ }^{2}$ Alimentary Pharmabiotic Centre, University College, Cork, Republic of Ireland
}

Epidemiological studies investigating associations between diet and cognitive function suggest a protective role for fish and fish-oil consumption in cognitive decline ${ }^{(1)}$ and depression ${ }^{(2)}$, particularly among elderly populations. As longevity increases and elderly subjects become an increasing proportion of populations worldwide, it is important to establish dietary components associated with healthy brain aging.

The aim of this study was to evaluate fish and fish-oil consumption in a sample of healthy, community-dwelling Irish adults and to explore the association between fish and fish oil consumption, and cognitive function and depression score.

Fish and fish product intakes, cognitive function and depressive symptoms were assessed among community-dwelling, elderly adults ( $n=172$; age 64-93 yrs) who participated in the ELDERMET project. Fish and fish product consumption was evaluated using a 147-item, validated, semi-quantitative Food Frequency Questionnaire, where fish intakes were categorized into the following groups; processed/fried fish, white fish, oily fish, shellfish and fish-oil supplements. The Mini Mental State Exam (MMsE) and Geriatric Depression Scale (GDS) were used to determine cognitive function and depressive symptoms, respectively.

Among the fish and fish-products assessed, white fish was consumed most frequently, by $84 \%$ of the group, with $72 \%$ of consumers complying with recommended intakes of one or more servings of white fish/wk. Seventy-six percent of the group consumed oily fish with $64 \%$ complying with recommendations. Shellfish consumption was lowest at $31 \%$. Consumption and compliance to recommended intakes was highest among females for all fish and fish-products, except for processed/fried fish consumption, which was higher among males. Fish-oils were consumed by $19 \%$ of the group, of which $55 \%$ were female.

No significant correlation was observed between white fish consumption and GDS (Pearson correlation coefficient $r=-0.007$; $P=0.97)$ and MMSE $(r=0.007 ; P=0.928)$. Similarly for oily fish, there was no association between GDS $(r=-0.072 ; P=0.427)$ and MMSE $>(r=0.052 ; P=0.497)$, indicating no positive effect of either white or oily fish consumption on improved depressive symptoms or cognitive function. However, an inverse association was found between fish-oil supplement consumption and GDS ( $r=-0.229$; $P=0.010$ ); suggesting an association between reduced depressive symptoms and increased fish-oil consumption. In addition, a trend for association between fish-oil consumption with MMse $(r=0.145 ; P=0.057)$ was also observed, whereby increasing fish-oil consumption was associated with a trend for improved cognitive function.

In conclusion, fish consumption was comparable with a nationally representative sample of healthy, elderly Irish individuals ${ }^{(3)}$. However gender differences indicate better compliance with guidelines among females. Further studies investigating the effect of fish-oil consumption on cognitive function and depressive symptoms in elderly groups are warranted. Fish consumption should be promoted in the elderly as it may prevent brain ageing processes and reduce cognitive decline.

1. Nugent et al. (2011) Prostaglandins Leukot Essent Fatty Acids 5, 287-91.

2. Bountziouka et al. (2009) J Aging Health 6, 864-80.

3. National Adult Nutrition Survey: Summary Report (2011) available online (http://www.iuna.net) 